\title{
Comparative Evaluation of a Modified Acetic Method for Extraction of Antioxidant Compounds from Black Beans (Phaseolus vulgaris) ${ }^{*}$
}

\author{
Jose F. Islas, Alfredo J. Dávalos-Balderas, Netzahualcóyotl Arroyo-Currás, Beiman G. Cano, \\ Paulina Galindo-Jacobo, Gustavo Guajardo-Salinas, Angel Gaytan-Ramos, Jorge E. Moreno-Cuevas
}

Laboratorio de Terapia Celular, Centro de Investigación y Transferencia en Salud (CITES)-Escuela de Medicina Ignacio A. Santos, Monterrey, México.

Email: jemoreno@itesm.mx

Received December $8^{\text {th }}$, 2011; revised February $10^{\text {th }}$, 2012; accepted February $17^{\text {th }}, 2012$

\begin{abstract}
Extraction of phenolic compounds from bean seed coats by acetic acid was evaluated and compared to conventional extraction with $80 \%$ methanol. Total phenols, flavonoids and free radical scavenging capacity were determined by colorimetric methods. Furthermore, qualitative flavonoid characterization was performed via HPLC diode array. The acetic acid extract contained $73.4 \pm 7 \mathrm{mg} / \mathrm{g}$ of sample expressed as gallic acid equivalents (GAE), and $41.3 \pm 4 \mathrm{mg} / \mathrm{g}$ as catechin equivalents (CE). The acetic acid extract (at $1 \mathrm{mg} / \mathrm{mL}$ ) showed over $89 \%$ efficiency to scavenge the 1,1 diphenyl2-picrylhydrazil $(\mathrm{DPPH})$ radical $\left(\mathrm{EC}_{50}=6.13 \mathrm{mg}\right.$ extract $/ \mathrm{mg}$ standard). Gallic acid, delphinidin-3-glycoside, petunidinglycoside, petunidin, malvidin-3-glycoside, myricetin-glycoside, quercitin-glycoside, saponin ( $\beta$-g), and 3-hydroxy-5, 7-megastigmadien-9-one were identified in both extracts. Additionally, kaempferol-3-glycoside was found in the acetic acid extract. Sequential acetic acid extractions indicated that the antiradical activity was about $90 \%$ in DPPH radical scavenging within the first $8 \mathrm{~h}$ of extraction, when compared to a $24 \mathrm{~h}$ extraction. In general, acetic acid offered a faster and more efficient approach for the extraction of phenolic antioxidants.
\end{abstract}

Keywords: Acetic Acid; Antioxidants; DPPH Scavenging; Extraction; Black Beans

\section{Introduction}

Common beans (Phaseolus vulgaris) and other leguminous are one of the milestones in most Latin-American and African diets [1]. Their high content of biologicallyactive chemical nutrients represents a great source of energy to the human organism, coming in part from the extended content of proteins and sugars, but also from phytochemical compounds such as flavonoids [2]. Flavonoids are known antioxidants that help protect the organism against cellular damage, reactive oxygen species (ROS), and other negative physiological effects [3,4]; moreover, their consumption-same as for other phytochemicals like anthocyanins, flavones, and flavanols has proven beneficial in the prevention of diseases like ischemic heart disease, stroke, and cancer [5-7]. Taking these nutritional sources into consideration, it is not surprising that many modern diets look towards an increase in the consumption of antioxidant compounds.

Over the years, diverse extraction methods have been

*The present work is a compilation of experimental results from a research project conducted between the years 2004-2005. used to successfully isolate antioxidants from various food matrices; however, a great majority of these methods are based on the application of mild, but yet toxic organic solvents like methanol and mixtures of water-ethanol [8-10]. The rationale behind the use of these methods is the fact that a polar solvent should interact with the highly polar functional groups bonded to the backbone of antioxidants. In this sense and for the particular case of black beans, an extraction method using 2:8 water in methanol $(\mathrm{v} / \mathrm{v})$ has been typically used to isolate polyphenolic compounds [11], along with HPLC-MS as the analytical method of choice to characterize the polyphenolic composition obtained. So far, HPLC-MS has been successfully used to separate and identify a large variety of flavonoids, anthocyanins and tannins present in black bean extracts [12].

It is the aim of the present study to present an effective and less toxic extraction method for polyphenolic compounds from black beans seed coats, hoping to obtain an extract with the potential to be used in clinical studies. The method proposed is based on the use of acetic acid as a replacing solvent for methanol. All experimental results are compared to a conventional extraction with $80 \%$ methanol. 


\section{Experimental Section}

\subsection{Preparation of Black Bean Extracts}

A black bean variety (Phaselous vulgaris) was planted in the Agriculture Experimental Station of the Instituto Tecnológico y de Estudios Superiores de Monterrey (ITESM), located in Nuevo León, Mexico. The ripe beans were harvested, air field dried, cleaned with sieves and air aspirated. Then, all clean beans were placed and stored in polyethylene bags. Separation of seed coats proceeded between two to three days after harvest.

The black beans were mechanically de-hulled using a decorticator equipped with abrasive disks (Nutana Machine Co., Saskatoon Canada). The objective of the milling procedure was to obtain two different fractions: seed coats and cotyledons. Grains were dried for 6 to 12 hours at $60^{\circ} \mathrm{C}$ in an air-forced convection oven. Optimum décor-tication was defined as the time required to remove between $13 \%$ to $15 \%$ of the grain weight in order to assure total removal of the hulls. The seed coat-rich material was separated from the cotyledon-rich material by air aspiration and sieving through a $2 \mathrm{~mm}$ opening sieve. At this point, seed coats were ready for the extraction procedure.

Two separate groups of dried bean seed coats were grinded in a commercial coffee grinder (Mr. Coffee, model IDS77) and placed in the following solvents: 5\% acetic acid in water $(\mathrm{v} / \mathrm{v})$ or $80 \%$ methanol in water $(\mathrm{v} / \mathrm{v})$. The blends were stirred for $24 \mathrm{~h}$ at a speed of $160 \mathrm{rpm}$ and at $45^{\circ} \mathrm{C}$ on an orbital Shaker (Gyromax 703, Amerex Instruments). The solution was then separated from the remaining solids by conventional filtration techniques.

The extracted liquid phase was concentrated first, and then evaporated to dryness with the aid of a rotary evaporator (R110, Brinkmann) operating at $75^{\circ} \mathrm{C}$ and under vacuum equivalent to $-70 \mathrm{kPa}$. The resulting extract was re-dissolved in $25 \mathrm{~mL}$ of physiological saline solution $(0.9 \% \mathrm{NaCl})$.

\subsection{Sample Solutions from Extract}

All experimental samples were prepared by taking 0.5 $\mathrm{mL}$ aliquots from each extract and independently mixing them with $0.5 \mathrm{~mL}$ of $1.2 \mathrm{M} \mathrm{HCl}$ in methanol (HPLC grade), to obtain a final concentration of $0.6 \mathrm{M} \mathrm{HCl}$ in $75 \%$ methanol. These samples were filtered through a PTFE microfilter ( $0.45 \mu \mathrm{m}$ pore size, Gelman, Ann Arbor, MI) prior to evaluation of antioxidant activity and HPLC analysis.

\subsection{Comparative Evaluation of Extracts}

\subsubsection{Critical Time of Extraction}

The two solvent systems were prepared as described before. To optimize extraction times, every eight hours the suspension of solvent (acetic acid and methanol) and seed coats was completely filtered in order to analyze the polyphenolic compounds extracted during that time lapse; later, the evaluation of antiradical activity by DPPH method [13] and the quantification of total phenolics via the Folin-Ciocalteu reagent [14] was performed. This procedure was repeated until no significant change was observed in the kinetics of reaction between the extract and both analytical evaluations.

\subsubsection{DPPH Assay}

The antiradical activity of the extract was calculated by means of the DPPH free radical activity assay. The DPPH concentration used was $0.25 \mathrm{mg} / \mathrm{mL}$ of $80 \%$ methanol. The evaluation of the reaction was conducted on 5 minutes intervals using an 8 well Spectrophotometer (Genesys 5, Spectronic, Milton Roy Co.). Aliquots of $100 \mu \mathrm{l}$ were taken from the different bean seeds extracts and mixed with $1 \mathrm{~mL}$ of the DPPH solution, and absorbance was recorded at $\lambda=515 \mathrm{~nm}$. Solutions of catechin $(0.288$ $\mathrm{mg} / \mathrm{mL})$ and gallic acid $(0.188 \mathrm{mg} / \mathrm{mL})$ were employed as reference standards to evaluate the antiradical power of the extracts. DPPH scavenging capacity was determined by plotting scavenging activity measured every 10 minutes until a total time of 1 hour was covered.

The $\mathrm{EC}_{50}$ index was calculated as the amount of antioxidant necessary to decrease the initial concentration of DPPH by $50 \%$. A calibration curve was constructed by measuring absorbance at $\lambda=515 \mathrm{~nm}$ vs. different concentrations of extract and a reference (trolox, gallic acid and catechin). The initial stock solution of samples and standards had a starting concentration of $1 \mathrm{mg} / \mathrm{mL}$, from which aliquots were taken to assess 10 fold decreases until a final concentration of $0.01 \mathrm{mg} / \mathrm{mL}$ was obtained.

\subsubsection{Determination of Total Phenolics}

Total phenolics were quantified by the Folin-Ciocalteu method. From a diluted sample of each extract prepared, $100 \mu \mathrm{l}$ were taken and mixed with $1 \mathrm{~mL}$ of the FolinCiocalteu reagent. The mixture was allowed to rest for 30 minutes before recording absorbance at $\lambda=750 \mathrm{~nm}$. Unmixed Folin-Ciocalteau solution was employed as a blank. Values were obtained after constructing a calibration curve by reacting the test reagent with different concentrations of gallic acid. The calibration curve included concentrations between $0.04 \mathrm{mg} / \mathrm{mL}-0.2 \mathrm{mg} / \mathrm{mL}$ of gallic acid $\left(R^{2}\right.$ $=0.996)$. Values are expressed as gallic acid equivalents (GAE).

\subsubsection{Determination of Total Flavonoids}

Content of total flavonoids was determined by means of a colorimetric method. Briefly, $250 \mu \mathrm{L}$ of diluted extract were mixed with $75 \mu \mathrm{L}$ of a $5 \% \mathrm{NaNO}_{2}$ solution, $150 \mu \mathrm{L}$ 
of a freshly prepared $10 \% \mathrm{AlCl}_{3}$ solution, and $500 \mu \mathrm{L}$ of $1 \mathrm{M} \mathrm{NaOH}$. The final volume was adjusted to $2.5 \mathrm{~mL}$ with deionized water. The mixture was allowed to rest for $5 \mathrm{~min}$, after which absorption was measured at $\lambda=510$ $\mathrm{nm}$. A mixed solution having no extract or standard was employed as a blank. The amount of total flavonoids was expressed as mg of catechin equivalents (CE)/g sample. The calibration curve of $\mathrm{CE}$ was constructed from solutions of pure catechin with conentrations ranging from $0.01-0.5 \mathrm{mg} / \mathrm{mL}\left(R^{2}=0.997\right)$.

\subsection{Chromatographic Analysis}

Separation and characterization of polyphenolic compounds was performed by colleagues at Centro de Biotecnología (ITESM, Monterrey, Mexico). The instrument employed was an HPLC device coupled to a diode array detector (2996 Waters Co., Milford, MA). Separation was performed on a reverse phase Waters Nova-Pak C18 column, $(3.9 \times 150 \mathrm{~mm}$, Waters Co. Milford, MA.), using $0.2 \mathrm{M} \mathrm{KH}_{2} \mathrm{PO}_{4}(\mathrm{pH} 2.4)$ as the mobile phase at a flow rate of $0.5 \mathrm{~mL} / \mathrm{min}$. Wavelength of $\lambda=280 \mathrm{~nm}$ was used for detection of phenolic acids and flavonoids.

To separate flavanols from other polyphenolic compounds, $1 \mathrm{~mL}$ samples of diluted extract were dissolved in a solution of $50 \%$ methanol in water and passed through a precondition C18 cartridge (Waters Sep Pak Cartridge, Milford MA). Then, $2 \mathrm{~mL}$ of methanol were passed through the same cartridge to recover bound compounds. The recovered solution was used for HPLC diode array analysis. The preconditioning of the cartridges was performed by washing with $4 \mathrm{~mL}$ of methanol followed by $10 \mathrm{~mL}$ of Nanopure water.

\section{Results and Discussion}

The use of acetic acid for extraction of antioxidants of phenolic nature is justified by its polarity, which is higher in comparative terms than that of other organic solvents [15]. Acetic acid is a weak acid that promotes the formation of hydrogen bonds while dissociated. This phenomenon plays a key role in the proposed extraction method, since formation of hydrogen bonds with oxygen-containing functional groups present in flavonoids enhances molecular interaction.

The acetic acid extraction procedure employed here demonstrated to be more efficient than other conventional methods, such as the 2:8 water-methanol system, by showing faster rates of neutralization of the DPP- radical after equivalent extraction times at $45^{\circ} \mathrm{C}$ [16]. Furthermore, the low concentration of acetic acid used during the extraction guaranteed the applicability of this method to produce extracts for posterior clinical applications, since acetic acid is not toxic in low concentrations and can be further neutralized by conventional acid-base titration.

Figure 1 presents a comparison between the antiradical activity of the acetic extract evaluated by DPPH test $v s$ that of two reference standards, catechin $(288 \mu \mathrm{g} / \mathrm{mL})$ and gallic acid $(188 \mu \mathrm{g} / \mathrm{mL})$, evaluated by the same method. Results showed close to $70 \%$ reduction of DPP- radical for the acetic extract at a concentration of $250 \mu \mathrm{g} / \mathrm{mL}$ after the first 5 minutes, versus $75 \%$ for gallic acid and 92\% for catechin.

On the other hand, Figure 2 shows a comparison between the behavior of the acetic extract $v s$ that of an extract obtained with the 2:8 water-methanol system. After completing three rounds of filtering, it was clear that close to $80 \%$ of the active compounds were removed during the first time point, thus explaining the lower antiradical activity at 24 hours. Acetic extract presents a faster rate of neutralization of DPP. radical than the more conven

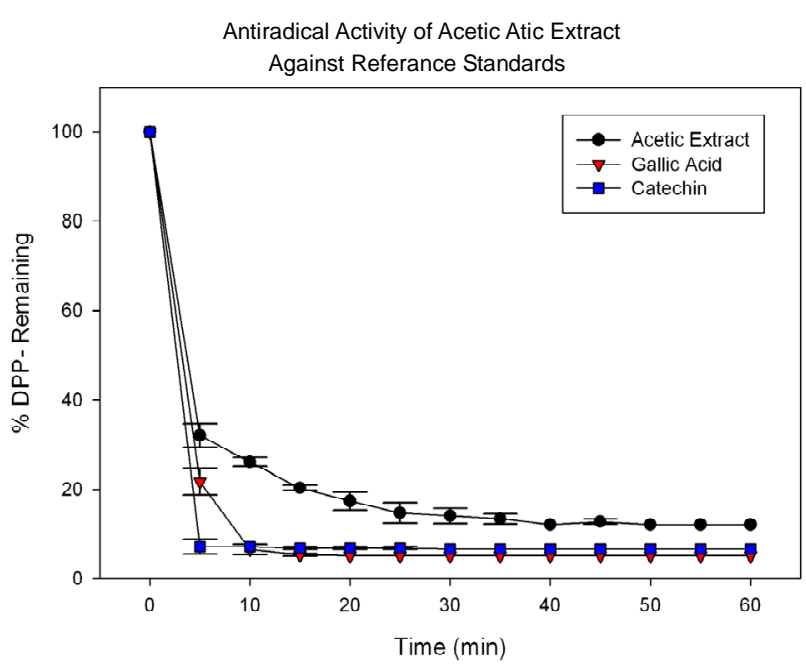

Figure 1. Response of acetic extract $(250 \mu \mathrm{g} / \mathrm{mL})$, gallic acid $(188 \mu \mathrm{g} / \mathrm{mL})$ and catechin $(288 \mu \mathrm{g} / \mathrm{mL})$ measured against DPP. radical.

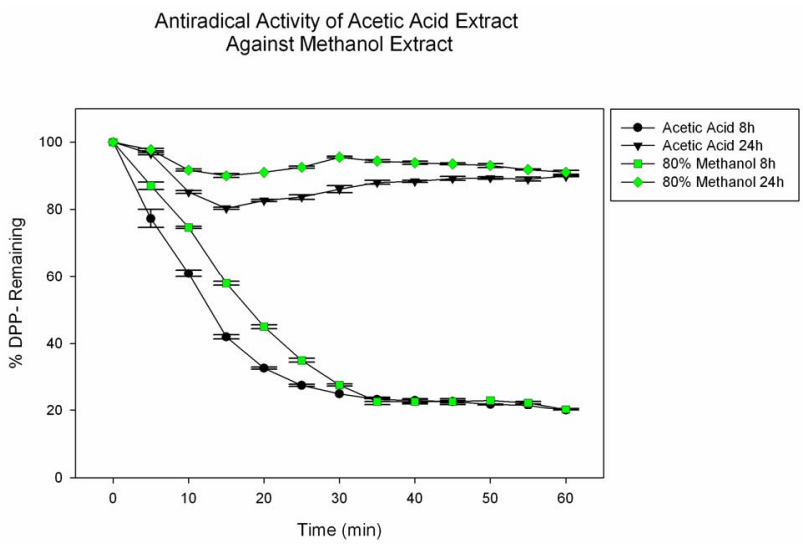

Figure 2. Response of acetic extract $(250 \mu \mathrm{g} / \mathrm{mL})$ and $80 \%$ methanol extract $(250 \mu \mathrm{g} / \mathrm{mL})$ measured against DPP. radical. 
tional extraction method under equal experimental conditions. From the data employed to construct Figure 1, a calculated $\mathrm{EC}_{50}$ of $6.13 \mathrm{mg}$ extract/mg DPPH was obtained for the equivalent concentration of antioxidants present in the acetic extract, with a correlation coefficient of $R^{2}=0.981$.

By using the Folin-Ciocalteu reagent, total content of phenolic compounds in the acetic extract was determined as $73.4 \mathrm{mg} \mathrm{GAE} / \mathrm{g}$ extract. In addition, a repetition of the same test but with catechin as standard gave $41.3 \mathrm{mg} \mathrm{CE} / \mathrm{g}$ extract. Suggesting that the acetic extract contains a high dosage of potent polyphenolic compounds with high antioxidant activity, when compared to methanol extracts. All experimental results confirm the idea that the proposed acetic acid extraction is highly effective to isolate and recover flavonoids from naturally occurring matrices, and that it does so with more efficiency than other extraction systems such as methanol [17] or acetone [11].

After the assessment of the extraction method capability was concluded, a composition analysis was carried out by HPLC-UV and HPLC-MS with the aid of colleagues from Centro de Biotecnología at ITESM, in Monterrey, Mexico, in order to reveal the nature of the antioxidant compounds present in the mixture. Figure 3 shows two representative chromatograms of the two extracts under consideration: acetic acid and $80 \%$ methanol.

The main observation is that a peak corresponding to the retention time of kaempferol-3-glycoside is observed only in the chromatogram of the acetic acid extract. This is important because kaempferol existing in both forms, glycosylated or not, is considered to be a potent antioxidant [18]. Characterization of all phenolic compounds present in the extracts was achieved by analysis of the retention times and by database matching. This procedure revealed the presence of 10 clearly characterized compounds: gallic acid; anthocyanins: delphinidin-3-glycoside, petunidin-glycoside, petunidin aglycone and malvidin-3-glycoside; flavonols: myricetin-glycoside, quercetin-glycoside and kaempferol-3-glycoside; saponins: saponin $(\beta$-g), and a rare megastigmane: 3-hydroxy-5,7megastigmadien-9-one. Table 1 shows retention times associated with each one of these compounds. These results were obtained in the form of a laboratory report and no information regarding the database consulted was stored.

The presence of some of these compounds has also been previously found in dried bean extracts [11,17] as well as in fruits $[18,19]$. Most of these compounds have demonstrated positive effects against chronic degenerative diseases. Malvidin has shown to play a protective role on hepatic diseases [20]. Kampferol has demonstrated to inhibit the release of proinflamatory metabolites IL-6, IL8, and $\mathrm{TNF}-\alpha$; therefore Kampferol is suitable for the treatment of allergic and inflammatory diseases [14,15]. More- over, related flavonols like Myricetin, Quercitin and Kampferol may act through antioxidant, prooxidant, antiestrogenic, cell signaling pathways modulation or mitochondrial toxicity to inhibit breast carcinogenesis $[1,20]$.

All experimental results presented so far suggest that the acetic acid system has an elevated capacity to extract antioxidant compounds. From an objective point of view, the composition of the acetic acid system (Figure 3(a)) seems to be close to the performance shown by the methanol system (Figure 3(b)). However, the antioxidant

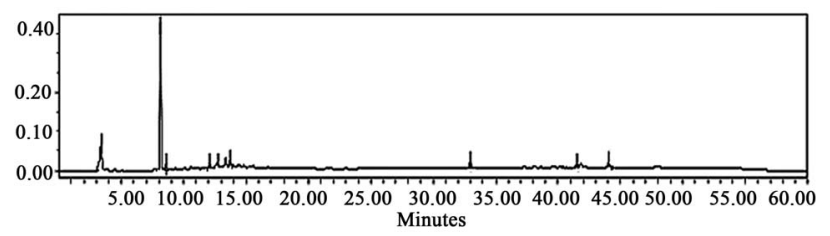

(a)

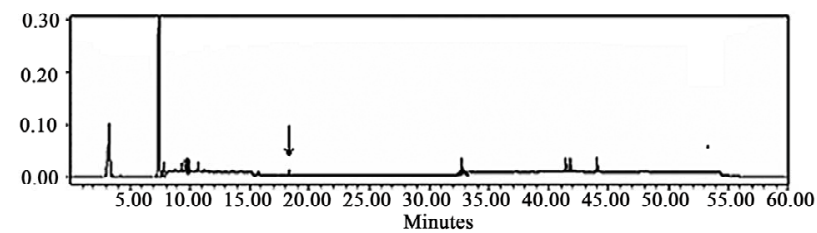

(b)

Figure 3. Low resolution HPLC-diode array chromatograms obtained from Centro de Biotecnología, corresponding to (a) the acetic acid extract; and (b) the $80 \%$ methanol extract. The black arrow on the chromatogram at the top suggests the presence of kaempferol-3-glycoside in the acetic acid extract, molecule considered to be a potent antioxidant [18].

Table 1. Names and retention times of antioxidant compounds identified in the acetic acid and $80 \%$ methanol extracts.

\begin{tabular}{ccc}
\hline \multirow{2}{*}{ Compound } & \multicolumn{2}{c}{ Retention Times $^{\mathrm{a}}$} \\
\cline { 2 - 3 } & Acetic acid & $80 \%$ methanol \\
\hline gallic acid & 10.029 & $10.01^{*}$ \\
delphinidin-3-glycoside & $12.02^{*}$ & 12.111 \\
petunidin-glycoside & $13.81^{*}$ & 12.242 \\
petunidin & $14.39^{*}$ & 12.229 \\
malvidin-3-glycoside & $14.61^{*}$ & 12.413 \\
myricetin-glycoside & $15.57^{*}$ & $14.27^{*}$ \\
quercetin-glycoside & $16.78^{*}$ & $17.39 *$ \\
kaempferol-3-glycoside & 18.246 & - \\
saponin ( $\beta$-g) & 32.788 & 33.071 \\
3-hydroxi-5,7-megastigmadien-9 one & 41.5 & 41.5 \\
\hline
\end{tabular}

${ }^{\mathrm{a}}$ Identification performed by database matching. *Identification obtained from high resolution chromatograms at higher concentration of extract (peaks not observable in Figure 3). 
activity tests presented in Figure 2 favors the acetic acid method in the neutralization of the DPP. radical. Moreover, methanol presents the drawback of being a toxic solvent.

The efficiency of extraction as a function of time was also evaluated. This was done with the objective of better understanding the ability of the different solvent systems to extract antioxidants at different times, and to integrate results so that the total extraction time could be reduced and optimized. These results demonstrate that the first 8 $\mathrm{h}$ are the most critical for the extraction of polyphenolic compounds. Eight hour extractions were able to decrease the concentration of DPP. active radical close to $80 \%$, compared to 24 hours, where most of the polyphenolic molecules have already been removed and the reduction of active DPP. radical is of only $20 \%$.

To further confirm these results, a total phenolic compound evaluation was done. From this evaluation, a composition graph (Figure 4) was drawn in order to compare the amount of antioxidants found in every extract at different times. As it can be observed, again more than $70 \%$ of the total phenolic compounds for the acetic acid system and over $60 \%$ for the methanol system were extracted during the first $8 \mathrm{~h}$.

\section{Conclusion}

Acetic acid, when diluted to a concentration of $5 \%$ acid in water, represents an alternative and efficient method for the extraction of antioxidants from black been seeds and other food matrices. The high antioxidant capacity of the extract obtained with this solvent was demonstrated by rapid kinetics of neutralization of DPP- radical, as well as by determination of the concentration of total phenolics present in the extract. In addition, acetic acid makes extraction of antioxidants not only highly efficient but also safe for animal testing (when compared to conventional methods involving the use of methanol or acetone).

\section{Total phenolic concentration}

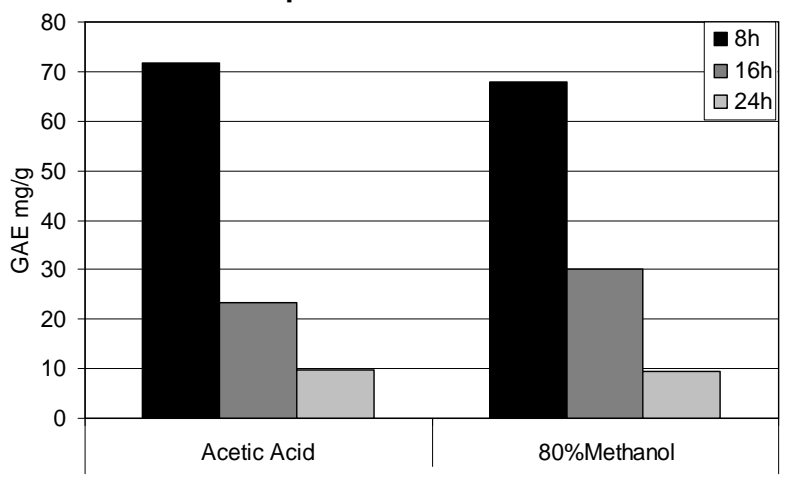

Figure 4. Effect of extraction time on the efficiency of phenolic extraction.

\section{Acknowledgements}

We thank Dr. Sergio O. Serna-Saldivar for providing us with laboratory-controlled beans, as well as for his valuable intellectual contribution to this work. Additionally, we would also like to thank Dr. Janet Gutierrez and Dr. Irasema Romo for performing all HPLC-MS and HPLCUV measurements for the present investigation. We thank Cátedra de Terapia Celular for providing the financial support that made this investigation possible.

\section{REFERENCES}

[1] S. H. Guzmán-Maldonado and O. Paredes-López, "Functional Products of Plants Indigineous to Latin America: Amaranth, Quinoa, Common Beans, and Botanicals, in Functional Foods: Biochemical \& Processing Aspects," Vol. 2, CRC Press, New York, 1998.

[2] K. A. Steinmetz and J. D. Potter, "Vegetables, Fruit, and Cancer Prevention: A Review," Journal of the American Diet Association, Vol. 96, No. 10, 1996, pp. 1027-1039. doi:10.1016/S0002-8223(96)00273-8

[3] C. A. Adebamowo, E. Cho, L. Sampson, M. B. Katan, D. Spiegelman, W. C. Willett and H. D. Holmes, "Dietary Flavonols and Flavonol-Rich Foods Intake and the Risk of Breast Cancer," International Journal of Cancer, Vol. 114, No. 4, 2005, pp. 628-633. doi:10.1002/ijc.20741

[4] C. Borek, "Antioxidant Health Effects of Aged Garlic Extract,” Journal of Nutrition, Vol. 131, 2001, pp. 10101015.

[5] A. R. Ness and J. W. Poulens, "Fruit, Vegetables, and Cardiovascular Disease: A Review,” International Journal of Epidemiology, Vol. 26, No. 1, 1997, pp. 1-13. doi:10.1093/ije/26.1.1

[6] D. X. Hou, "Potential Mechanisms of Cancer Chemoprevention by Anthocyanins," Current Molecular Medicine, Vol. 3, No. 2, 2003, pp. 149-159. doi:10.2174/1566524033361555

[7] T. Hirvonen, J. Virtamo, P. Korhonen, D. Albanes and P. Pietinen, "Flavonol and Flavone Intake and the Risk of Cancer in Male Smokers," Cancer Causes and Control, Vol. 12, 2001, pp. 789-796. doi:10.1023/A:1012232008016

[8] Y. L. Lee, C. C. Weng and J. L. Mau, “Antioxidant Properties of Ethanolic and Hot Water Extracts from the Rhizome of Curcuma Aromatica," Journal of Food Biochemistry, Vol. 31, No. 6, 2007, pp. 757-771. doi:10.1111/j.1745-4514.2007.00143.x

[9] A. A. Adedapo, F. O. Jimoh, S. Koduru, A. J. Afolayan and P. J. Masika, “Antibacterial and Antioxidant Properties of the Metanol Extracts of the Leaves and Stems of Calpurnia Aurea," BMC Complementary and Alternative Medicine, Vol. 8, 2008, pp. 53-61. doi:10.1186/1472-6882-8-53

[10] A. Ghasemzadeh, H. Z. E. Jaafar and A. Rahmat, "Effects of Solvent Type on Phenolics and Flavonoids Content 
and Antioxidant Activities in Two Varieties of Young Ginger Extracts,” Journal of Medicinal Plants Research, Vol. 5, No. 7, pp. 1147-1154.

[11] X. Aparicio-Fernandez, G. G. Yousef, G. Lorca-Pina, E. Mejia and M. A. Lila, "Characterization of Polyphenolics in the Seed Coat of Black Jamapa Bean (Phaseolus vulgaris L.)," Journal of Agricultural and Food Chemistry, Vol. 53, No. 11, 2005, pp. 4615-4622.

doi:10.1021/jf0478020

[12] D. Heimler, P. Vignolini, M. G. Dini and A. Romani, "Rapid Tests to Assess the Antioxidant Activity of Phaseolus vulgaris L. Dry Beans," Journal of Agricultural and Food Chemistry, Vol. 53, 2005, pp. 3053-3056. doi:10.1021/jf049001r

[13] O. P. Sharma and T. K. Bhat, "DPPH Antioxidant Assay Revisited,” Analytical Methods, Vol. 113, No. 4, 2009, pp. 1202-1205.

[14] E. Roura, C. Andrés-Lacueva, R. Estruch and R. M. Lamuela-Reventós, “Total Polyphenol Intake Estimated by a Modified Folin-Ciocalteau Assay of Urine,” Clinical Chemistry, Vol. 52, No. 4, 2006, pp. 749-752. doi:10.1373/clinchem.2005.063628

[15] J. A. Byers, "Solvent Polarity and Miscibility Data," 2003. http://www.chemical-ecology.net/java/solvents.htm

[16] Z. Mohammedi and F. Atik, "Impact of Solvent Extraction Type on Total Polyphenols Content and Biological
Activity from Tamarix Aphylla (L.) Karst,” International Journal of Pharma and Bio Sciences, Vol. 2, No. 1, 2011, pp. 609-615.

[17] C. W. Beninger and G. L. Hosfield, “Antioxidant Activity of Extracts, Condensed Tannin Fractions, and Pure Flavonoids from Phaseolus vulgaris L. Seed Coat Color Genotypes," Journal of Agricultural and Food Chemistry, Vol. 51, No. 27, 2003, pp. 7879-7883. doi:10.1021/jf0304324

[18] H. Wang, M. G. Nair, G. M. Strasburg, A. M. Booren and J. I. Gray, "Antioxidant Polyphenols from Tart Cherries (Prunus cerasus)," Journal of Agricultural and Food Chemistry, Vol. 47, No. 3, 1999, pp. 840-844. doi:10.1021/jf980936f

[19] K. Robards, X. Li, M. Antolovich and S. Boyd, "Characterization of Citrus by Chromatographic Analysis of Flavonoids," Journal of the Science of Food and Agriculture, Vol. 75, No. 1, 1997, pp. 87-101. doi:10.1002/(SICI)1097-0010(199709)75:1<87::AID-JSF A846>3.0.CO;2-B

[20] Y. P. Jang, J. Zhou, K. Nakanishi and J. R. Sparrow, "Anthocyanins Protect against A2E Photooxidation and Membrane Permeabilization in Retinal Pigment Epithelial Cells," Photochemistry and Photobiology, Vol. 81, No. 3, pp. 2005, pp. 529-533. 